\title{
Nobel Lecture: A confrontation with infinity*
}

\author{
Gerard 't Hooft ${ }^{\dagger}$ \\ Institute for Theoretical Physics, University of Utrecht, 3584 CC Utrecht, The Netherlands
}

\section{APPETIZER}

Early attempts at constructing realistic models for the weak interaction between elementary particles were offset by the emergence of infinite, hence meaningless, expressions when one tried to derive the radiative corrections. When models based on gauge theories with Higgs mechanism were discovered to be renormalizable, the bothersome infinities disappeared-they cancelled out. If this success seemed to be due to mathematical sorcery, it may be of interest to explain the physical insights on which it is actually based.

\section{INTRODUCTION}

It is the highest possible honor for a scientist in my field to be standing here and give this lecture. It is difficult to express how thankful I am, not only to the Nobel Committee and to the Royal Swedish Academy of Sciences, but also to the numerous fellow physicists and friends who considered our work to be of such importance that we should be nominated to receive this Prize. In this lecture I intend to reflect on the efforts that were needed to tame the gauge theories, the reasons for our successes at this point, and the lessons to be learned. I realize the dangers of that. Often in the past, progress was made precisely because lessons from the past were being ignored. Be that as it may, I nevertheless think these lessons are of great importance, and if researchers in the future should choose to ignore them, they must know what they are doing.

When I entered the field of elementary particle physics, no precise theory for the weak interactions existed (for an account of the historical developments, see, for instance, Crease and Mann, 1986). It was said that any theory one attempted to write down was nonrenormalizable. What was meant by that? In practice, what it meant was that when one tried to compute corrections to scattering amplitudes, physically impossible expressions were encountered. The result of the computations appeared to imply that these amplitudes should be infinite. Typically, integrals of the following form were found:

$$
\int d^{4} k \frac{\operatorname{Pol}\left(k_{\mu}\right)}{\left(k^{2}+m^{2}\right)\left[(k+q)^{2}+m^{2}\right]}=\infty,
$$

\footnotetext{
*The 1999 Nobel Prize in Physics was shared by Gerard 't Hooft and Martinus J. G. Veltman. This lecture is the text of Professor 't Hooft's address on the occasion of the award.

${ }^{\dagger}$ Electronic address: g.thooft@fys.ruu.nl
}

where $\operatorname{Pol}\left(k_{\mu}\right)$ stands for some polynomial in the integration variables $k_{\mu}$. Physically, this must be nonsense. If, in whatever model calculation, the effects due to some obscure secondary phenomenon appear to be infinitely strong, one knows what this means: the so-called secondary effect is not as innocent as it might have appeared-it must have been represented incorrectly in the model; one has to improve the model by paying special attention to the features that were at first thought to be negligible. The infinities in the weak-interaction theories were due to interactions from virtual particles at extremely high energies. High energy also means high momentum, and in quantum mechanics this means that the waves associated with these particles have very short wavelengths. One had to conclude that the shortdistance structure of the existing theories was too poorly understood.

Short distance scales and short time intervals entered into theories of physics first when Newton and Leibniz introduced the notion of differentiation. In describing the motion of planets and moons, one had to consider some small time interval $\Delta t$ and the displacement $\Delta \vec{x}$ of the object during this time interval [see Fig. 1(a)]. The crucial observation was that, in the limit $\Delta t \rightarrow 0$, the ratio

$$
\frac{\Delta \vec{x}}{\Delta t}=\vec{v}
$$

makes sense, and we call it "velocity." In fact, one may again take the ratio of the velocity change $\Delta \vec{v}$ during such a small time interval $\Delta t$, and again the ratio

$$
\frac{\Delta \vec{v}}{\Delta t}=\vec{a}
$$

exists in the limit $\Delta t \rightarrow 0$; we call it "acceleration." Their big discovery was that it makes sense to write equations relating accelerations, velocities, and positions, and that in the limit where $\Delta t$ goes to zero, you get good models describing the motion of celestial bodies [Fig. 1(c)]. The mathematics of differential equations grew out of this, and nowadays it is such a central element in theoretical physics that we often do not realize how important and how nontrivial these observations actually were. In modern theories of physics we send distances and time inter-

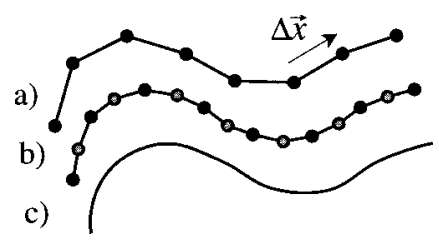

FIG. 1. Differentiation. 
vals to zero all the time, also in multidimensional field theories, assuming that the philosophy of differential equations applies. But occasionally it may happen that everything goes wrong. The limits that we thought to be familiar with do not appear to exist. The behavior of our model at the very tiniest time and distance scales then has to be reexamined.

Infinite integrals in particle theory were not new. They had been encountered many times before, and in some theories it was understood how to deal with them (see, for instance, Pais, 1986). What had to be done was called "renormalization." Imagine a particle such as an electron to be something like a little sphere, of radius $R$ and mass $m_{\text {bare }}$. Now attach an electric charge to this particle, of an amount $Q$. The electric-field energy would be

$$
U=\frac{Q^{2}}{8 \pi R},
$$

and, according to Einstein's special theory of relativity, this would represent an extra amount of mass, $U / c^{2}$, where $c$ is the speed of light. Particle plus field would carry a mass equal to

$$
m_{\text {phys }}=m_{\text {bare }}+\frac{Q^{2}}{8 \pi c^{2} R} .
$$

It is this mass, called "physical mass," that an experimenter would measure if the particle were subject to Newton's law, $\vec{F}=m_{\text {phys }} \vec{a}$. What is alarming about this effect is that the mass correction diverges to infinity when the radius $R$ of our particle is sent to zero. But we want $R$ to be zero, because if $R$ were finite it would be difficult to take into account that forces acting on the particle must be transmitted by a speed less than that of light, as is demanded by Einstein's theory of special relativity. If the particle were deformable, it would not be truly elementary. Therefore, finite-size particles cannot serve as a good basis for a theory of elementary objects.

In addition, there is an effect that alters the electric charge of the particle. This effect is called "vacuum polarization." During extremely short time intervals, quantum fluctuations cause the creation and subsequent annihilation of particle-antiparticle pairs. If these particles carry electric charges, the charges whose signs are opposite to our particle in question tend to move towards it, and this way they tend to neutralize it. Although this effect is usually quite small, there is a tendency of the vacuum to "screen" the charge of our particle. This screening effect implies that a particle whose charge is $Q_{\text {bare }}$ looks like a particle with a smaller charge $Q_{\text {phys }}$ when viewed at some distance. The relation between $Q_{\text {bare }}$ and $Q_{\text {phys }}$ again depends on $R$, and, as was the case for the mass of the particle, the charge renormalization also tends to infinity as the radius $R$ is sent to zero (even though the effect is usually rather small at finite $R$ ).

It was already in the first half of the 20th century that physicists realized the following. The only properties of a particle such as an electron that we ever measure in an experiment are the physical mass $m_{\text {phys }}$ and the physical charge $Q_{\text {phys }}$. So, the procedure we have to apply is that we should take the limit where $R$ is sent to zero while $m_{\text {phys }}$ and $Q_{\text {phys }}$ are kept fixed. Whatever happens to the bare mass $m_{\text {bare }}$ and the bare charge $Q_{\text {bare }}$ in that limit is irrelevant, since these quantities can never be measured directly.

Of course, there is a danger in this argument. If, in Eq. (2.5), we send $R$ to zero while keeping $m_{\text {phys }}$ fixed, we notice that $m_{\text {bare }}$ tends to minus infinity. Can theories in which particles have negative mass be nevertheless stable? The answer is no, but fortunately, Eq. (2.3) is replaced by a different equation in a quantized theory. $m_{\text {bare }}$ tends to zero, not minus infinity.

\section{THE RENORMALIZATION GROUP}

The modern way to discuss the relevance of the smalldistance structure is by performing scale transformations, using the renormalization group (Wilson and Kogut, 1974; Politzer, 1974), and we can illustrate this again by considering the equation of motion of the planets. Assume that we took definite time intervals $\Delta t$, finding equations for the displacements $\Delta x$. Imagine that we wish to take the limit $\Delta t \rightarrow 0$ very carefully. We may decide first to divide all $\Delta t$ 's and all $\Delta x$ 's by 2 [see Fig. 1(b)]. We observe that, if the original intervals are already sufficiently small, the new results of a calculation will be very nearly the same as the old ones. This is because, during small time intervals, planets and moons move along small sections of their orbits, which are very nearly straight lines. If they had been moving exactly along straight lines, the division by 2 would have made no difference at all. Planets move along straight lines if no force acts on them. The reason why differential equations were at all successful for planets is that we may ignore the effects of the forces (the "interactions") when time and space intervals are taken to be very small.

In quantized field theories for elementary particles, we have learned how to do the same thing. We reconsider the system of interacting particles at very short time and distance scales. If at sufficiently tiny scales the interactions among the particles may be ignored, then we can understand how to take the limits where these scales go all the way to zero. Since then the interactions may be ignored, all particles move undisturbedly at these scales, and so the physics is then understood. Such theories can be based on a sound mathematical footing - we understand how to do calculations by approximating space and time as being divided into finite sections and intervals and taking the limits in the end.

So, what is the situation here? Do the mutual interactions among elementary particles vanish at sufficiently tiny scales? Here is the surprise that physicists had to learn to cope with: they do not.

Many theories indeed show very bad behavior at short distances. A simple prototype of these is the so-called chiral model (see a description in Lee, 1972, pp. 60-67). 
In such a model, a multicomponent scalar field is introduced which obeys a constraint: its total length is assumed to be fixed,

$$
\sum_{i}\left|\phi_{i}\right|^{2}=R^{2}=\text { fixed. }
$$

At large distance scales, the effects of this constraint are mild, as the quantum fluctuations are small compared to $R$. At small distance scales, however, the quantum fluctuations are large compared to $R$, and hence the nonlinear effects of the constraint are felt much more strongly there. As a consequence, such a theory has large interactions at small distance scales and vice versa. Therefore, at infinitesimally small distance scales, such a theory is ill-defined, and the model is unsuitable for an accurate description of elementary particles. Other examples of models with bad small-distance behavior are the old four-fermion interaction model for the weak interactions and most attempts at making a quantum version of Einstein's gravity theory.

But some specially designed models are not so bad. Examples are: a model with spinless particles whose fields $\phi$ interact only through a term of the form $\lambda \phi^{4}$ in the Lagrangian, and a model in which charged particles interact through Maxwell's equations (quantum electrodynamics, QED). In general, we choose the distance scale to be a parameter called $1 / \mu$. A scale transformation by a factor of 2 amounts to adding $\ln 2$ to $\ln \mu$, and if the distance scale is $\Delta x$, then

$$
\frac{\mu d}{d \mu} \Delta x=-\Delta x \text {. }
$$

During the 1960's, it was found that in all theories existing at the time, the interaction parameters, being either the coefficient $\lambda$ for $\lambda \phi^{4}$ theory, or the coefficient $e^{2}$ in quantum electrodynamics for electrons with charge $e$, the variation with $\mu$ is a positive function (Gross, 1997), called the $\beta$ function:

$$
\frac{\mu d}{d \mu} \lambda=\beta(\lambda)>0,
$$

so, comparing this with Eq. (3.2), $\lambda$ is seen to increase if $\Delta x$ decreases.

In the very special models that we just mentioned, the function $\beta(\lambda)$ behaves as $\lambda^{2}$ when $\lambda$ is small, which is so small that the coupling only varies very slightly as we go from one scale to the next. This implies that, although there are still interactions, no matter how small the scales at which we look, these interactions are not very harmful, and a consequence of this is that these theories are "renormalizable." If we apply the perturbation expansion for small $\lambda$ then, term by term, the expansion coefficients are uniquely defined, and we might be seduced into believing that there are no real problems with these theories.

However, many experts in these matters were worried indeed, and for good reason: If $\beta$ is positive, then there will be a scale where the coupling strength among particles diverges. The solution to Eq. (3.3) is [see Fig. 2(a)]
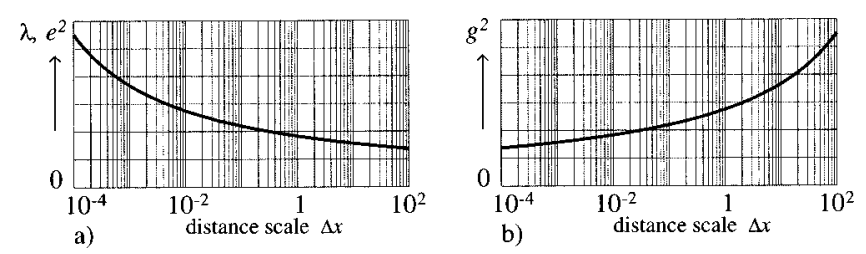

FIG. 2. Scaling of the coupling strength as the distance scale varies, (a) for $\lambda \phi^{4}$ theories and QED, (b) for Yang-Mills theories.

$$
\lambda(\mu)=1 /\left(C-\beta_{2} \ln \mu\right), \quad \text { if } \beta(\lambda)=\beta_{2} \lambda^{2},
$$

where $C$ is an integration constant, $C=1 / \lambda(1)$ if $\lambda(1)$ is $\lambda$ measured at the scale $\mu=1$. We see that at scales $\mu$ $=\mathcal{O}\left[\exp \left(1 / \beta_{2} \lambda(1)\right)\right]$, the coupling explodes. Since for small $\lambda(1)$ this is exponentially far away, the problem is not noticed in the perturbative formulation of the theory, but it was recognized that if, as in physically realistic theories, $\lambda$ is taken to be not very small, there is real trouble at some definite scale. And so it was not so crazy to conclude that these quantum field theories were sick and that other methods should be sought for describing particle theories.

I was never afflicted with such worries for a very simple reason. Back in 1971, I carried out my own calculations of the scaling properties of field theories, and the first theory I tried was Yang-Mills theory. My finding was, when phrased in modern notation, that for these theories,

$$
\beta\left(g^{2}\right)=C g^{4}+\mathcal{O}\left(g^{6}\right) \quad \text { with } C<0
$$

if the number of fermion species is less than 11 [for $\mathrm{SU}(2)$ ] or $16 \frac{1}{2}$ [for $\mathrm{SU}(3)$ ]. The calculation, which was alluded to in my first paper on the massive Yang-Mills theory ('t Hooft, 1971) was technically delicate but conceptually not very difficult. I could not possibly imagine what treasure I had here or that none of the experts knew that $\beta$ could be negative; they had always limited themselves to studying only scalar field theories and quantum electrodynamics, where $\beta$ is positive.

\section{THE STANDARD MODEL}

If we were to confront the infinities in our calculations for the weak-interaction processes, we had to face the challenge of identifying a model for the weak interaction that shows the correct intertwining with the electromagnetic force at large distance scales but is sufficiently weakly interacting at small distances. The resolution here was to make use of spontaneous symmetry breaking. The mass generation mechanism discussed here should, strictly speaking, not be regarded as spontaneous symmetry breaking, since in these theories the vacuum does not break the gauge symmetry. "Hidden symmetry" is a better phrase (Coleman, 1975). We simply refer to this mechanism as the "Higgs mechanism." We use a field with a quartic self-interaction but with a negative mass term, so that its energetically favored value is nonvanishing. The fact that such fields can be used to generate massive vector particles was known but 
not used extensively in the literature. Also the fact that one could construct reasonable models for the weak interaction along these lines was known. These models, however, were thought to be inelegant, and the fact that they were the unique solution to our problems was not realized.

Not only did the newly revived models predict hitherto unknown channels for the weak interaction, they also predicted a new scalar particle, the Higgs boson (Higgs, 1964a, 1964b, 1966) The new weak interaction, the so-called neutral-current interaction, could be confirmed experimentally within a few years, but as of this writing, the Higgs boson is still fugitive. Some researchers suspect that it does not exist at all. Now if this were true then this would be tantamount to identifying the Higgs field with a chiral field - a field with a fixed length. We could also say that this corresponds to the limiting case in which the Higgs mass was sent to infinity. An infinite-mass particle cannot be produced, so it can be declared to be absent. But as we explained before, chiral theories have bad small-distance behavior. We can also say that the interaction strength at small distances is proportional to the Higgs mass; if that would be taken to be infinite then we would have landed in a situation where the small-distance behavior was out of control. Such models simply do not work. Perhaps experimentalists will not succeed in producing and detecting Higgs particles, but this then would imply that entirely new theories must be found to account for the small-distance structure. Candidates for such theories have been proposed. They seem to be inelegant at present, but of course that could be due to our present limited understanding, who knows? New theories would necessarily imply the existence of many presently unknown particle species, and experimenters would be delighted to detect and study such objects. We cannot lose here. Either the Higgs particle or other particles must be waiting there to be discovered, probably fairly soon (seem, for instance, Accomando et al., 1998, and Zerwas, 1999).

To the strong interactions, the same philosophy applies, but the outcome of our reasoning is very different. The good scaling behavior of pure gauge theories [see Fig. 2(b)] allows us to construct a model in which the interactions at large distance scales is unboundedly strong, yet it decreases to zero (though only logarithmically) at small distances. Such a theory may describe the binding forces between quarks. It was found that these forces obtain a constant strength at arbitrarily large distances, where Coulomb forces would have decreased with an inverse square law. Quantum chromodynamics, a Yang-Mills theory with gauge group SU(3), could therefore serve as a theory for the strong interactions. It is the only allowed model in which the coupling strength is large but nevertheless the small-distance structure is under control.

The weak force, in contrast, decreases exponentially as the distance between weakly interacting objects becomes large. Thus gauge theory allows us to construct models with physically acceptable behavior at short dis-

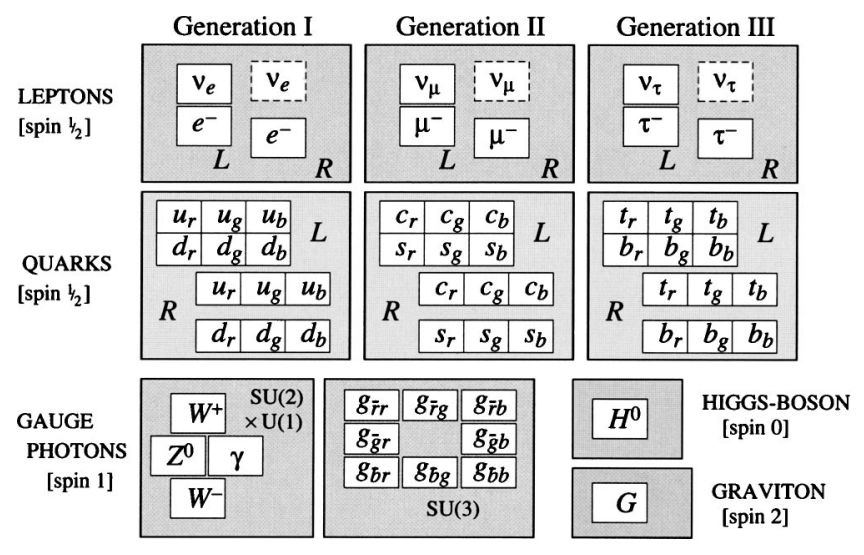

FIG. 3. The Standard Model.

tances, while the forces at large distances may vary in any of the following three distinctive ways:

(i) The force may drop exponentially fast, as in the weak interaction;

(ii) The force may drop according to an inversesquare law, as in electromagnetism, or

(iii) The force may tend towards a constant, as in the strong interactions.

The Standard Model is the most accurate model describing nature as it is known today. It is built exactly in accordance with the rules sketched in Fig. 3. Our philosophy is always that the experimentally obtained information about the elementary particles refers to their large-distance behavior. The small-distance structure of the theory is then postulated to be as regular as is possible without violating principles such as strict obedience of causality and Lorentz invariance. Not only do such models allow us to calculate their implications accurately, it appears that Nature really is built this way. In some sense, this result appears to be too good to be true. We shall shortly explain our reasons to suspect the existence of many kinds of particles and forces that could not yet be included in the Standard Model, and that the small-distance structure of the Standard Model does require modification.

\section{FUTURE COLLIDERS}

Theoreticians are most eager to derive all they want to know about the structures at smaller distances using pure thought and fundamental principles. Unfortunately, our present insights are hopelessly insufficient, and all we have are some wild speculations. Surely, the future of this field still largely depends on the insights to be obtained from new experiments.

The present experiments at the Large Electron Positron Collider (LEP) at CERN are coming to a close. They have provided us with impressive precision measurements that not only gave a beautiful confirmation of the Standard Model, but also allowed us to extrapolate to higher energies, which means that we were allowed a glimpse of structures at the smallest distance ranges yet accessible. The most remarkable result is that the structures there appear to be smooth; new interactions could 
not be detected, which indicates that the mass of the Higgs particle is not so large, a welcome stimulus for further experimental efforts to detect it.

In the immediate future we may expect interesting new experimental results first from the Tevatron Collider at Fermilab, near Chicago, and then from the Large Hadron Collider (LHC) at CERN, both of which will devote much effort to finding the still elusive Higgs particle. Who will be first depends on what the Higgs mass will turn out to be, as well as other not yet precisely known properties of the Higgs. Detailed analysis of what we know at present indicates that Fermilab has a sizable chance at detecting the Higgs first, and the LHC almost certainly will not only detect these particles, but also measure many of their properties, such as their masses, with high precision. If supersymmetric particles exist, LHC will also be in a good position to be able to detect these, in measurements that are expected to begin shortly after 2005 .

These machines, which will dissolve structures never seen before, however, also have their limits. They stop exactly at the point where our theories become highly interesting, and the need will be felt to proceed further. As before, the options are either to use hadrons such as protons colliding against antiprotons, which has the advantage that, due to their high mass, higher energies can be reached, or alternatively to use leptons, such as $e^{+}$ colliding against $e^{-}$, which has the advantage that these objects are much more pointlike, and their signals are more suitable for precision experiments (Ellis, 1999) Of course, one should do both. A more ambitious plan is to collide muons, $\mu^{+}$against $\mu^{-}$, since these are leptons with high masses, but this will require numerous technical hurdles to be overcome. Boosting the energies to ever-increasing values requires such machines to be very large. In particular the high-energy electrons will be hard to force into circular orbits, which is why design studies of the future accelerators tend to take the form of straight lines, not circles. These linear accelerators have the interesting feature that they could be extended to larger sizes in the more distant future.

My hope is that efforts and enthusiasm to design and construct such machines in the future will not diminish. As much international cooperation as possible is called for. A sympathetic proposal (Zichichi; 1999) is called ELOISATRON, a machine in which the highest conceivable energies should be reached in a gigantically large circular tunnel. It could lead to a hundredfold improvement of our spatial resolution. What worries me, however, is that in practice one group, one nation, takes an initiative and then asks other groups and nations to join, not so much in the planning, but rather in financing the whole thing. It is clear to me that the best international collaborations arise when all partners are involved from the very earliest stages of the development onwards. The best successes will come from those institutions that are the closest approximations to what could be called "world machines." CERN claims to be a world machine, and indeed as such this laboratory has been, and hopefully will continue to be, extremely successful.
Unfortunately, it still has an E in its name. This E should be made as meaningless as the $\mathrm{N}$ (after all, the physics studied at CERN has long ago ceased to be nuclear, it is subnuclear now). I would not propose to change the name, but to keep the name CERN only to commemorate its rich history.

\section{BEYOND THE STANDARD MODEL}

Other, equally interesting large scientific enterprises will be multinational by their very nature: plans are underway to construct neutrino beams that go right through the earth to be detected at the exit point, where it may be established how subtle oscillations due to their small mass values may have caused transitions from one type into another. Making world machines will not imply that competition will be eliminated; the competition, however, will not be between nations, but rather between the different collaborations who use different machines and different approaches towards physics questions.

The most interesting and important experiments are those of which we cannot guess the outcome reliably. This is exactly the case for the LHC experiments that are planned for the near future. What we do know is that the Standard Model, as it stands today, cannot be entirely correct, in spite of the fact that the interactions stay weak at ultrashort distance scales. Weakness of the interactions at short distances is not enough; we also insist that there be a certain amount of stability. Let us use the metaphor of the planets in their orbits once again. We insisted that, during extremely short time intervals, the effects of the forces acting on the planets have hardly any effect on their velocities, so that they move approximately in straight lines. In our present theories, it is as if at short time intervals several extremely strong forces act on the planets, but, for some reason, they all but balance out. The net force is so weak that only after long time intervals, days, weeks, months, the velocity change of the planets become apparent. In such a situation, however, a reason must be found as to why the forces at short time scales balance out. The way things are for the elementary particles, at present, is that the forces balance out just by accident. It would be an inexplicable accident, and as no other examples of such accidents are known in Nature, at least not of this magnitude, it is reasonable to suspect that the true shortdistance structure is not exactly as described in the Standard Model, but that there are more particles and forces involved, whose nature is as yet unclear. These particles and forces are arranged in a new symmetry pattern, and it is this symmetry that explains why the short-distance forces balance out.

It is generally agreed that the most attractive scenario is one involving "supersymmetry," a symmetry relating fermionic particles, whose spin is an integer plus onehalf, and bosonic particles, which have integral spin. (Supersymmetry has a vast literature. See, for instance, the collection of papers in Ferrara, 1987.) It is the only symmetry that can be made to do the required job in the presence of the scalar fields that provide the Higgs 
mechanism, in an environment where all elementary particles interact weakly. However, when the interactions do eventually become strong then there are other scenarios. In that case, the objects playing the role of Higgs particles may be not elementary objects but composites, similar to the so-called Cooper pairs of bound electrons that perform a Higgs mechanism in ultracool solid substances, leading to superconductivity. Just because such phenomena are well known in physics, this is a scenario that cannot easily be dismissed. But, since there is no evidence at present of a new strong interaction domain at the $\mathrm{TeV}$ scale, the bound-state Higgs theory is not favored by most investigators.

One of the problems with the supersymmetry scenario is the supersymmetry breaking mechanism. Since at the distance scale where experiments are done at present no supersymmetry has been detected, the symmetry is broken. It is assumed that the breaking is "soft," which means that its effects are seen only at large distances, and only at the tiniest possible distance scales is the symmetry realized. Mathematically, this is a possibility, but there is as yet no plausible physical explanation of this situation. The only explanation can come from a theory at even smaller distance scales, where the gravitational force comes into play.

Until the early 1980's the most promising model for the gravitational force was a supersymmetric variety of gravity: supergravity (Ferrara, 1987, Vol. 2) It appeared that the infinities that were insurmountable in a plain gravity theory would be overcome in supergravity. Curiously, however, the infinities appeared to be controlled by the enhanced symmetry and not by an improved small-distance structure of the theory. Newton's constant, even if controlled by a dilaton field, still is dimensionful in such theories, with consequently uncontrolled strong interactions in the small-distance domain. As the small-distance structure of the theory was not understood, it appeared to be almost impossible to draw conclusions from the theory that could shed further light on empirical features of our world.

An era followed with even wilder speculations concerning the nature of the gravitational force. By far the most popular and potentially powerful theory is that of the superstrings (see, for instance, Polchinski, 1998). The theory started out by presenting particles as made up of (either closed or open) pieces of string. Fermions living on the string provide it with a supersymmetric pattern, which may be the origin of the approximate supersymmetry that we need in our theories. It is now understood that only in a perturbative formulation do particles look like strings. In a nonperturbative formalism there seems to be a need not only of strings but also of higherdimensional substances such as membranes. But what exactly is the perturbation expansion in question? It is not the approximation that can be used at the shortest infinitesimal distances. Instead, the shortest distances seem to be linked to the largest distances by means of duality relations. Just because superstrings are also held responsible for the gravitational force, they cause curva- ture of space and time to such an extent that it appears to be futile to consider distances short compared to the Planck scale.

According to superstring theory, it is a natural and inevitable aspect of the theory that distance scales shorter than the Planck scale cannot be properly addressed, and we should not worry about it. When outsiders or sometimes colleagues from unrelated branches of physics attack superstring theory, I come to its defense. The ideas are very powerful and promising. But when among friends, I have this critical note. As string theory makes heavy use of differential equations it is clear that some sort of continuity is counted on. We should attempt to find an improved short-distance formulation of theories of this sort, if only to justify the use of differential equations or even functional integrals.

Rather than regarding the above as criticism against existing theories, one should take our observations as indications of where to search for further improvements. Emphasizing the flaws of the existing constructions is the best way to find new and improved procedures. Only in this way can we hope to achieve theories that allow us to explain the observed structures of the Standard Model and to arrive at more new predictions, so that we can tell our experimental friends where to search for new particles and forces.

\section{REFERENCES}

Accomando, E., et al., 1998, Phys. Rep. 299, 1.

Coleman, S., 1975, "Secret Symmetries," in Laws of Hadronic Matter, edited by A. Zichichi (Academic, New York/ London).

Crease, R. P., and C. C. Mann, 1986, The Second Creation: Makers of the Revolution in Twentieth-century Physics (Macmillan, New York).

Ellis, J., 1999, "Possible Accelerators at CERN beyond the LHC," preprint CERN-TH/99-350, hep-ph/9911440.

Englert, F., and R. Brout, 1964, Phys. Rev. Lett. 13, 508.

Ferrara, S., 1987, Ed., Supersymmetry, Vols. 1 and 2 (NorthHolland, Amsterdam).

Gross, D. J., 1997, in The Rise of the Standard Model (Cambridge University, Cambridge), p. 199.

Higgs, P. W., 1964a, Phys. Lett. 12, 132.

Higgs, P. W., 1964b, Phys. Rev. Lett. 13, 321.

Higgs, P. W., 1966, Phys. Rev. 145, 1156.

Lee, B. W., 1972, Chiral Dynamics (Gordon and Breach, New York), pp. 60-67.

Pais, A., 1986, Inward Bound: Of Matter and Forces in the Physical World (Oxford University, London).

Polchinski, J., 1998, String Theory, Vol. 1, An Introduction to the Bosonic String, Cambridge Monographs on Mathematical Physics, edited by P. V. Landshoff et al. (Cambridge University, Cambridge).

Politzer, H. D., 1974, Phys. Rep., Phys. Lett. 14C, 129.

't Hooft, G., 1971, Nucl. Phys. B 35, 167.

Wilson, K. G., and J. Kogut, 1974, Phys. Rep., Phys. Lett. 12C, 75.

Zerwas, P. M., 1999, "Physics with an $e^{+} e^{-}$linear collider at 
high luminosity," Cargèse lectures 1999, preprint DESY 99178.

Zichichi, A., 1999, "Fifty years of subnuclear physics: from past to future and the ELN project," in Highlights of Sub- nuclear Physics: 50 Years Later: Proceedings of the International School of Subnuclear Physics, edited by A. Zichichi (World Scientific, Singapore and River Edge, London), p. 161. 